\title{
Relationship between Intensity and Reaction Time in Normal-Hearing Infants and Adults
}

\author{
Lori J. Leibold and Lynne A. Werner
}

\begin{abstract}
Objective: Reaction time (RT) to sound is known to be related to loudness in adult listeners. The purpose of this study was to determine whether infants' RT to sound decreases systematically with intensity as it does in adults.
\end{abstract}

Design: RT was measured for 24 6- to 9-mo-old infants and 11 19- to 26-yr-old adults. All participants were normal hearing, naïve listeners. The stimuli consisted of 4000 and $1000 \mathrm{~Hz}$ pure tones presented to the right ear through an insert earphone. Stimulus intensities ranged in $10 \mathrm{~dB}$ steps from 40 to $80 \mathrm{~dB}$ SPL for adults and 50 to $90 \mathrm{~dB}$ SPL for infants. Infant responses consisted of a head turn toward a reinforcer whereas adults responded by raising their hand. An additional three adults responded with a head turn. RT was defined as the time between the onset of the tone and an observer's button press indicating that a response had occurred. RT was corrected for the observer's reaction time and averaged over three to five repetitions at each level to obtain the mean reaction time (MRT) for each subject, frequency, and level.

Results: MRT decreased with increasing intensity in both infants and adults. An examination of the MRT-intensity functions suggests that the infant functions may be steeper than those of adults, although considerable variability exists between listeners.

Conclusions: RT holds potential as a measure of loudness in infants. Whether differences in the MRT-intensity slopes exist between infants and adults is unclear. Future investigations using methods to reduce the variability of RT measurements are needed to examine potential slope differences further.

(Ear \& Hearing 2002;23;92-97)

Although pitch and timbre have been studied in infants (e.g., Clarkson, Clifton 1985; Clarkson, Clifton, Perris 1988), loudness has not. The development of loudness perception is interesting for a number of reasons. From a research perspective, understanding how loudness perception develops may provide clues about the mechanisms underlying developmental changes in auditory sensitivity. Clinically, the perception of loudness has received a

University of Washington, Seattle, Washington. great deal of recent attention. Many current hearing aid fitting strategies aim to "normalize" loudness growth in individuals with hearing impairment who demonstrate a more-rapid-than-normal growth of loudness (e.g., Cox, 1995). What is "normal" for infants is unclear. Given the role that abnormal loudness growth plays in the diagnosis and management of hearing loss, it is very important to know whether loudness growth is different in infants and why. However, infants do not express how loud something sounds to them in a readily interpretable way.

In adults, the relationship between loudness and intensity has been measured in various ways, including scaling and matching. The loudness of a sound is a function of many factors including duration, intensity, bandwidth and frequency. However, for adult listeners, loudness has been shown to increase as a power function of sound pressure with an exponent of approximately 0.6 (e.g., Scharf, 1978).

Investigators reporting developmental changes in masked thresholds have suggested that infants have a reduced growth of neural excitation with increasing intensity (Schneider \& Trehub, 1992; Schneider, Trehub, Morrongiello, \& Thorpe, 1989). This hypothesis would predict a slower growth of loudness in infants compared with adults.

Bartoshuk (1964) examined the relationship between cardiac acceleration and intensity for a 1000 $\mathrm{Hz}$ pure tone in newborns. The logarithm of cardiac acceleration was reported to increase linearly with stimulus level with an exponent of 0.53 . This result has never been replicated, and to our knowledge, no further studies of infant loudness perception are reported in the literature.

Studies of older children suggest that loudness perception is mature by 4 to $5 \mathrm{yr}$. Collins and Gescheider (1989) used both absolute magnitude estimation (AME) and cross modality-matching (CMM) techniques to compare the perception of loudness in adults and 4- to 7-yr-old children. Results indicated no significant differences between child and adult loudness growth functions. More recently, Serpanos and Gravel (2000) reported mature loudness growth functions in 4- to 12-yr-old normal-hearing children using a modified CMM technique. Additionally, the investigators measured 
significantly steeper loudness growth functions in children with sensorineural hearing loss, consistent with CMM being a reliable measure of loudness growth both in normal-hearing children as young as $4 \mathrm{yr}$ and hearing-impaired children as young as $6 \mathrm{yr}$. Similarly, studies of loudness discomfort levels (LDLs) in children over the age of 7 indicate no significant differences compared with adult listeners (Kawell, Kopun, \& Stelmachowitz, 1988; Stuart, Durieux-Smith, \& Stenstrom, 1991).

Traditional methods of measuring loudness require sophisticated responses infants cannot make. One alternative might be reaction time (RT) to sound. Chocholle (1940) was the first to demonstrate that $\mathrm{RT}$ varies inversely with loudness. That is, the interval between the onset of the stimulus and the listener's behavioral response becomes shorter as the stimulus becomes more intense. Furthermore, he demonstrated that equal-loudness contours obtained using RT data are very similar to those obtained using loudness-balancing methods (but see Kohfeld, Santee, \& Wallace, 1981).

Several researchers have used the RT paradigm to study loudness in human adults (e.g., Humes \& Ahlstrom, 1984; Kohfeld et al., 1981; Marshall \& Brandt, 1980; Seitz \& Rakerd, 1997). In particular, Humes and Ahlstrom (1984) obtained both RT-intensity and loudness-growth functions based on magnitude estimation from normal-hearing, trained adult listeners. Frequencies of 1000, 2000, and 4000 $\mathrm{Hz}$ were tested. Correlations between slopes of the RT-intensity and loudness-growth functions were found to be comparable to the test-retest correlations for the loudness-growth functions at 2000 and $4000 \mathrm{~Hz}$. The authors suggested that a RT paradigm might be used to indirectly estimate loudness. More recently, investigators have examined the relationship between RT-intensity functions and subjective loudness measures in subjects with both normal hearing and sensorineural hearing loss with recruitment (Seitz \& Rakerd, 1997). Their findings suggest that RT is a feasible method to measure loudness both in normal-hearing and hearing-impaired listeners.

The purpose of the current investigation was to examine the feasibility of using a RT paradigm to study loudness perception in infants and adults. Although studies have examined the relationship between RT and intensity in trained adults, no studies have examined the relationship in untrained adults or infants. Because infants cannot respond by pressing a button, head turns were measured. Infant head turns are commonly used to investigate infants' hearing both in the clinic and the laboratory. They are appealing behaviors to measure because they are easily measured in infants as young as 5 to 6 mo (Moore, Wilson, \& Thompson, 1977). Interrater reliability of head-turn judgments is quite high, on the order of 0.95 (e.g., Olsho, 1984). As Luce (1986) points out, however, the motor, sensory, and attentional factors that influence RT to sound are complex. Given that infants cannot be instructed and that their responses cannot be tightly controlled, it is possible that no reliable relationship between their RT and sound intensity can be identified. Should such a relationship be demonstrated, though, it would provide another tool for investigating early human auditory development.

\section{MethoD}

\section{Subjects}

Data were collected from 24 6- to 9-mo-old infants and 11 19- to 26-yr-old adults. The average age at the initial testing session was $34.1 \mathrm{wk}$ ( $\mathrm{SD}=3.4 \mathrm{wk}$ ) for infants and $22.5 \mathrm{yr}(\mathrm{SD}=2.3 \mathrm{yr})$ for adults. The data from 12 additional infants were excluded from analysis. Eleven infants were unable to complete training and, one infant was excluded due to experimenter error. Participant selection criteria were 1) no risk factors for hearing loss as assessed by parental or self-report, 2) no more than two episodes of otitis media, 3) not under treatment for otitis media within the prior week, and 4) healthy on the test date. Additionally, screening tympanometry was performed on every subject at each session. Peak admittance of at least $0.2 \mathrm{mmhos}$ at a pressure between -200 and 50 daPa was required to pass the screening.

No participant had more than 2 yr of musical training nor had participated in any other psychoacoustical experiments.

\section{Stimuli and Procedure}

The primary test frequency was $4000 \mathrm{~Hz}$, a frequency at which 6-mo-old's absolute thresholds are relatively mature (reviewed by Werner \& Marean, 1996) and at which significant correlations between loudness growth and RT-intensity slopes have been observed for adults (Humes \& Ahlstrom, 1984). For participants who completed testing at $4000 \mathrm{~Hz}$ quickly enough, testing at $1000 \mathrm{~Hz}$ was subsequently attempted. Infants' absolute thresholds are less mature at $1000 \mathrm{~Hz}$ than $4000 \mathrm{~Hz}$ (Werner \& Marean, 1996), and the correlation between sensitivity and reaction time was of interest. Of course, any differences observed between responses to 4000 $\mathrm{Hz}$ and those to $1000 \mathrm{~Hz}$ would have to be viewed cautiously because frequency is confounded with test order in this design. However, this approach 
ensured that data were obtained at the frequency of primary interest.

All tones were digitally generated, attenuated (Wilserics PATT programmable attenuator), filtered (Kemo VBF dual variable filter), amplified (locallybuilt amplifier), and presented to the right ear through an ER1 insert earphone for 500 msec. A rise/fall time of $16 \mathrm{msec}$ was used. Tone intensities ranged in $10 \mathrm{~dB}$ steps from 50 to $90 \mathrm{~dB}$ SPL for infants and 40 to $80 \mathrm{~dB}$ SPL for adults. The higher test intensities were selected for infants to compensate for the elevation in their detection thresholds compared with adults (reviewed by Werner and Marean, 1996). The selected levels correspond approximately to a range of 40 to $80 \mathrm{~dB}$ average sensation level for both groups. An examination of response rate as a function of level demonstrated that infants responded at least $85 \%$ of the time at each level. These response rates are consistent with the asymptotic levels for detection reported by Bargones, Werner, \& Marean (1995), suggesting that the infants were performing maximally at each level.

Data were collected in separate blocks of trials for each frequency. Adult subjects were tested in a 1-hr visit. Infants were tested in two 1-hr visits occurring within a 2 -wk period. Data were collected initially at $4000 \mathrm{~Hz}$. If testing was successful in the first session, data were collected in the next session at 1000 $\mathrm{Hz}$. If not successful, $4000 \mathrm{~Hz}$ was retested during the second session.

Testing was conducted in a double-walled, soundattenuating room (IAC). Infants were tested sitting on their parent's lab. An assistant sat to the left and in front of the parent and infant. The assistant and the parent both wore circumaural headphones, which delivered masking sounds. The headphones were worn to prevent the adults from hearing the tones and influencing the infant's response.

Infants were conditioned to make head turns toward their right when the tone was heard. An observer outside the booth initiated a trial and a tone was presented. When the observer judged that a response had occurred, she pressed a button to end the trial and initiate reinforcement. Reinforcement was the activation and illumination of a mechanical toy. If no response occurred within $4 \mathrm{sec}$ of presenting the stimulus, the trial ended and no reinforcement was given.

Each session began with a training phase. The level of the tone was $60 \mathrm{~dB}$ SPL, and infants were required to respond to the tone on three consecutive trials to complete the phase. Those infants completing the training phase proceeded to testing. The test phase consisted of six random presentations of each of the 5 intensity levels, for a total of 30 trials per session. The observer, who was blind to the intensity of the signal, pushed a button if a response was observed. To guard against anticipation effects in the absence of catch trials, a random interstimulus interval was used. For both infants and adults, the minimum interval between a response and the presentation of the next stimulus was 4 sec. For adults, the maximum interstimulus interval was $10 \mathrm{sec}$. For infants, the maximum interstimulus interval was typically $10 \mathrm{sec}$, although occasionally a longer interval was used if the infant was moving excessively during the pretrial period.

The procedure used to test adults was the same as that used for infants. However, adult participants were alone in the booth throughout testing and were asked to raise their hand when they heard a sound. Again, an observer outside the booth pressed the button when a response was observed to end the trial and provide feedback in the form of illumination of the mechanical toy.

To determine differences in the time required to generate a hand raise and a head turn (at least for adults), we required three additional adults to generate both head turn and hand raise responses in different sessions. Results for these participants were collected for the $4000 \mathrm{~Hz}$ condition only.

If no response occurred on a trial, the trial was excluded from analysis. Sessions were considered successful only if the participant completed all 30 test trials and responded to a minimum of four of six trials at each intensity level. This exclusion ensured that all intensities were equally audible, to guard against the possibility that an infant with hearing loss slipped through the screening. This exclusion is particularly important because measures of loudness growth are affected by sensorineural hearing loss (e.g., Hellman \& Meiselman, 1990). For adults, 11 of 11 sessions were successful at both 1000 and $4000 \mathrm{~Hz}$. For infants, 13 of 24 sessions were successful at $4000 \mathrm{~Hz}$, and 10 of 13 sessions were successful at $1000 \mathrm{~Hz}$.

Two adults were tested as those described above using hand raise responses at $4000 \mathrm{~Hz}$. In subsequent sessions they were tested multiple times until their performance reached an asymptotic level. In these sessions the two adults were instructed to push a button as soon as they heard a tone. The observer's contribution to the measured adult RT was estimated by calculating the difference between the geometric mean RT obtained from the two trained adults in the initial testing session using hand raises and the final testing session using button presses. The mean difference across these two subjects (173.8 msec) was subsequently subtracted from all RTs to remove the component due to the observer's RT. 


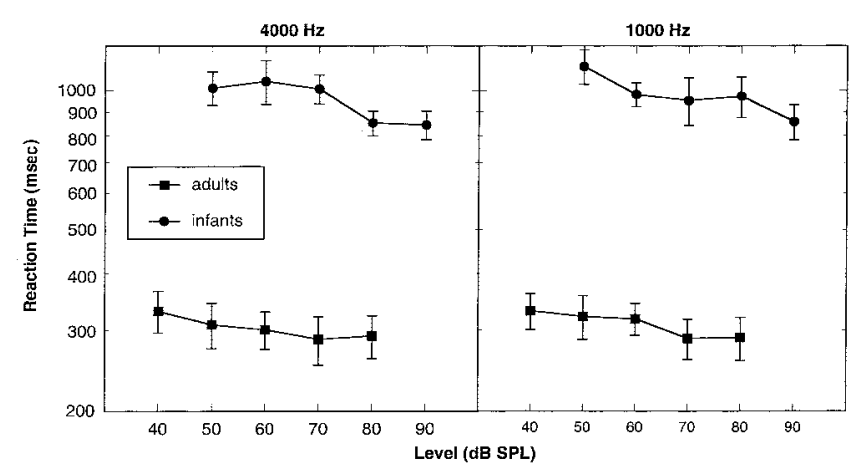

Figure 1. Average MRTs as a function of level for the two age groups at 4000 and $1000 \mathrm{~Hz}$. Error bars indicate \pm 1 standard error of the mean.

Previous investigations have adopted several methods for dealing with reaction time outliers to counteract the effects of processes such as inattention and signal anticipations (see Ratcliff, 1993). In the current paradigm, false short RTs are not expected to be frequent because listeners did not know exactly when a signal might occur. However, false long RTs are expected to pose some difficulty. Thus, our adopted approach was to remove the highest RT at each level for all subjects. Additionally, one remaining infant $\mathrm{RT}$ at $1000 \mathrm{~Hz}$ was greater than 3 sec and was also removed. The data analyzed were the mean reaction times (MRT) for each subject at each level and frequency.

\section{Results}

Average MRT as a function of intensity is shown in Figure 1 for infants and adults at 4000 and 1000 $\mathrm{Hz}$. Average MRT decreased with increasing level for infants and adults at both frequencies. The rate of MRT change appears to be greater for infants than adults.

To test the differences in MRT across levels, a repeated measures Level $\times$ Frequency analysis of variance was conducted on the logarithm of MRT for each age group. Only the eight infants completing testing successfully at both frequencies were included in the repeated measures analysis of variance.

For adults, the MRTs obtained at 4000 and 1000 $\mathrm{Hz}$ were not significantly different $[\mathrm{F}(1,7)=1.214$, $p=0.307]$. However, a significant main effect of intensity level on MRT was found $[\mathrm{F}(4,28)=5.633$, $p=0.002$ ]. That is, adult MRTs became significantly smaller with increasing intensity. No interaction between intensity level and frequency was observed $[\mathrm{F}(4,28)=2.037, p=0.116]$.

Similar findings were observed for infants. Again, no significant differences in the MRTs of infants

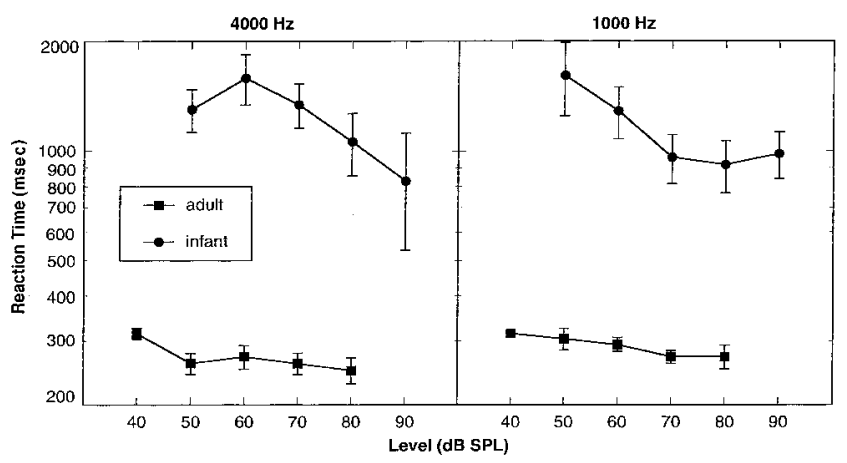

Figure 2. Examples of individual RT data for one infant and one adult at each frequency. Error bars represent \pm 1 standard error of the mean.

were found between 1000 and $4000 \mathrm{~Hz}[\mathrm{~F}(1,10)=$ $0.104, p=0.754]$. Infants, like adults, demonstrated a significant main effect of intensity level on MRT [F $(4,40)=21.728, p<0.000]$, with MRT decreasing significantly with increasing level.* No significant interaction between frequency and level was found $[\mathrm{F}(4,40)=0.459, p=0.765]$.

No direct comparison between the MRTs of infants of adults was performed because the assumption of homogeneity of variance was violated. However, large infant-adult differences in MRT appear to be present. The difference between hand-raises and head-turns is a potential explanation for the age-difference in MRT. However, for the three adult subjects who responded with head turns, MRTs ranged from 251 to $415 \mathrm{msec}$. These MRTs are similar to those observed from the main adult group who responded with hand raises.

Most participants demonstrated decreasing RTs with increasing level, although individual functions were not always monotonic. Examples of individual data for one adult and one infant at each frequency are shown in Figure 2.

The slope of the MRT-intensity function was calculated for each subject using a least squares solution of $\log (\mathrm{MRT})=\log \mathrm{b}+\mathrm{a} \log \mathrm{P}$, where $\mathrm{b}$ is a constant, a is the slope of the line, and $\mathrm{P}$ is sound pressure. Ten of 13 infants at $4 \mathrm{kHz}, 7$ of 10 infants at $1 \mathrm{kHz}$, and 10 of 11 adults at each frequency produced MRT-intensity functions with negative slopes. For functions with $\mathrm{R}^{2}$ of at least 0.40 , the mean slopes for adults were $-0.040(\mathrm{SD}=0.009)$ and $-0.050(\mathrm{SD}=0.026$ ) at 4000 and $1000 \mathrm{~Hz}$, respectively. Similarly, the three adults required to produce head turn responses produced slopes of $-0.046,-0.057$, and -0.029 and the two trained adult listeners tested with button press responses produced slopes of -0.051 and -0.047 . In contrast,

*The significant main effect of intensity was obtained, even when no outliers were excluded. 
mean slopes for the infants were -0.067 (SD $=$ $0.037)$ and $-0.098(\mathrm{SD}=0.048)$ at 4000 and 1000 $\mathrm{Hz}$, respectively. $\dagger$

Although the slopes were quite variable across subjects, and only half of the infants provided stable fits, these data suggest that MRT-intensity functions of individual infants may be steeper than those of adults.

\section{Discussion}

The main result of this study is that MRT decreased systematically with increasing intensity both in adults and infants. Because the RT paradigm has been used successfully to study loudness in human adults (e.g., Seitz \& Rakerd, 1997), the current findings suggest that RT measures offer promise in the study of infant loudness perception as well.

As in published reports where RT generally ranges from 200 to $400 \mathrm{msec}$ in individual listeners (e.g., Humes \& Ahlstrom, 1984), adult MRTs ranged from 200 to $500 \mathrm{msec}$ for both hand raise and head turn responses. Infant MRTs were much higher, ranging from 500 to 2000 msec. Because RT reflects both sensory and nonsensory components, it is not clear whether the age differences in $\mathrm{RT}$ reflect age differences in sound processing time or response generation. Certainly infant responses could be delayed due to a lack of attention or motivation to respond quickly. In addition, infants execute motor responses more slowly than do adults (reviewed by Schmidt \& Lee, 1999).

Given the complexity of RT and the potential for between-infant variability in each component, it is remarkable that their MRT can be shown to change reliably with intensity. On the other hand, immaturity of nonsensory components of RT may reduce the utility of RT for studying loudness development. For example, could the apparent slope difference between infants and adults be explained by infants' longer nonsensory RT? In general, the longer the nonsensory component of RT, the shallower the RT-intensity function slope (reviewed by Kohfeld et al., 1981).

Our adult listeners' average MRT-intensity function slopes were -0.040 and -0.050 at 4000 and $1000 \mathrm{~Hz}$, respectively. Humes and Ahlstrom (1984) reported average slopes of -0.055 and -0.049 . The infant slopes appear much higher than the adult

\footnotetext{
$\dagger$ Following Luce (1986), we subsequently calculated least-squares estimates of Piéron's law, MRT $=r_{o}+k P \beta$, where $r_{o}$ is the "nonsensory" component, $\mathrm{k}$ is a constant, $\mathrm{P}$ is sound pressure, and $\beta$ is the power exponent, for all subjects. Although the range for $\beta$ was very large $(-0.05$ to -0.98$)$, the average values were consistent with those summarized by Luce (1986).
}

slopes. This finding was surprising, given that the nonsensory component of RT for the infants is likely greater than that of adults. However, due to the considerable variability in infant slopes as well as the fact that over half of the infant functions were not well fit by a power function, the true nature of the age difference in slopes remains unclear. The fact that average infant RT varied systematically with intensity suggests it is worthwhile to identify methods that reduce individual variability.

In summary, the observation that RT generally decreased with increasing intensity, for both infants and adults, suggests that studying auditory development using this approach is feasible. Should slope differences between infants and adults be confirmed, future investigations using the RT paradigm would still be needed to establish that a difference in loudness growth is involved. For example, manipulations known to affect loudness in adults should have similar effects on infants' RT-intensity slopes.

\section{ACKNOWLeDGMents}

The authors would like to thank Nicole Holmer, Ellen Wilcox and Bonnie McTaggert for their assistance in data collection and subject scheduling. This work was supported by the National Institutes of Health (DC00396-15).

Address for correspondence: Lori Leibold, M.Sc., University of Washington, Department of Speech and Hearing Sciences, 1417 NE 42nd Street, Seattle, WA 98105-6246.

Received November 7, 2001; accepted November 9, 2001

\section{REFERENCES}

Bargones, J. Y., Werner, L. A., \& Marean, G. C. (1995). Infant psychometric functions for detection: Mechanisms of immature sensitivity. Journal of the Acoustical Society of America, 98, 99-111.

Bartoshuk, A. K. (1964). Human neonatal cardiac responses to sound: A power function. Psychonomic Science, 1, 151-152.

Chochelle, R. (1940). Variation des temps de reaction auditifs en fonction de l'intensite a diverses frequencies. Annee Psychologique, 41, 65-124.

Clarkson, M. G., \& Clifton, R. K. (1985). Infant pitch perception: Evidence for responding to pitch categories and the missing fundamental. Journal of the Acoustical Society of America, 77, 1521-1528.

Clarkson, M. G., Clifton, R. K., \& Perris, E. E. (1988). Infant timbre perception: Discrimination of spectral envelopes. Perception and Psychophysics, 43, 15-20.

Collins, A. A., \& Gescheider, G. A. (1989). The measurement of loudness in individual children and adults by absolute magnitude estimation and cross-modality matching. Journal of the Acoustical Society of America, 85, 2012-2021.

Cox, R. M. (1995). Using loudness data for hearing aid selection: The IHAFF approach. The Hearing Journal, 48(2), 10-44.

Hellman, R. P., \& Meiselman, C. H. (1990). Loudness relations for individuals and groups in normal and impaired hearing. Journal of the Acoustical Society of America, 88, 2596-2606.

Humes, L. E., \& Ahlstrom, J. B. (1984). Relation between reaction time and loudness. Journal of Speech and Hearing Research, 27, 306-310. 
Kawell, M. E., Kopun, J. G., \& Stelmachowitz, P. G. (1988). Loudness discomfort levels in children. Ear and Hearing, 9, 133-136.

Kohfeld, D. L., Santee, J. L., \& Wallace, N. D. (1981). Loudness and reaction time: I. Perception and Psychophysics, 29, 535-549.

Luce, R. D. (1986). Detection paradigms. In R. D. Luce (Ed.), Response Times: Their Role in Inferring Elementary Mental Organization (pp. 47-94). New York: Oxford University Press.

Marshall, L., \& Brandt, J. F. (1980). The relationship between loudness and reaction time in normal hearing listeners. Acta Otolaryngologica, 90, 244-249.

Moore, J. M., Wilson, W. R., \& Thompson, G. (1977). Visual reinforcement of head-turn responses in infants under 12 months of age. Journal of Speech and Hearing Disorders, 42, 328-334.

Olsho, L. W. (1984). Infant frequency discrimination. Infant Behavior and Development, 7, 27-35.

Ratcliff, R. (1993). Methods for dealing with reaction time outliers. Psychological Bulletin, 114, 510-532.

Scharf, B. (1978). Loudness. In E. C. Caterette \& M. P. Friedman (Eds.), Handbook of Perception (pp. 187-242). New York: Academic Press.
Schmidt, R. A., \& Lee, T. D. (1999). Motor Control and Learning: A Behavioral Emphasis. Champaign, IL: Human Kinetics.

Schneider, B. A., \& Trehub, S. E. (1992). Sources of developmental change in auditory sensitivity. In L. A. Werner \& E. W. Rubel (Eds.), Developmental Psychoacoustics (pp. 3-46). Washington, DC: American Psychological Association.

Schneider, B. A., Trehub, S. E., Morrongiello, B. A., \& Thorpe, L. A. (1989). Developmental changes in masked thresholds. Journal of the Acoustical Society of America, 86, 1733-1742.

Seitz, P. F., \& Rakerd, B. (1997). Auditory stimulus intensity and reaction time in listeners with longstanding SNHL. Ear and Hearing, 18, 502-512.

Serpanos, Y. C., \& Gravel, J. S. (2000). Assessing growth of loudness in children by cross-modality matching. Journal of the American Academy of Audiology, 11, 190-202.

Stuart, A., Durieux-Smith, A., \& Stenstrom, R. (1991). Probe tube microphone measures of loudness discomfort levels in children. Ear and Hearing, 12, 140-143.

Werner, L. A., \& Marean, G. C. (1996). Human Auditory Development. Boulder, CO: Westview Press. 